\title{
Mechanical and Thermal Properties of Poly(vinyl alcohol) Crosslinked by Borax
}

\author{
Hiroshi Ochiai, Sachiko Fukushima, Miyuki Fujikawa, \\ and Hitoshi Yamamura \\ Department of Chemistry, Faculty of Science, \\ Hiroshima University, Hiroshima, Japan.
}

(Received June 26, 1975)

\begin{abstract}
KEY WORDS Mechanical Property / Thermal Property / Poly(vinyl alcohol) / Borax / Crosslinking / Glass Transition /
\end{abstract}

The results of investigations of the mechanical and thermal properties of poly(vinyl alcohol) crosslinked by borax are reported.

It has been known that an aqueous solution of PVA is readily gelled by the addition of borax and that this gelation is caused by the network formation of borate chelate ions between the borate ions and hydroxyl groups on adjacent polymer strands. ${ }^{1-6}$ The crosslinking reaction is as follow:<smiles>CC(O)CC(O)CC(C)O[B-]1(OC(C)CC(C)O)OC(C)CC1[PH3+]</smiles>

Furthermore, for this system the crosslink density is easily controlled by changing the concentrations of borate ion, and a firm and transparent film can be also obtained by drying the gels. On crosslinking, one can expect two separate influences on the molecular organizations of PVA chain: ${ }^{7}$ a crosslinking effect resulting in immobilization of chain segments at the chelate points between the $\mathrm{OH}$ groups and the borate ions, and a copolymer effect resulting in depression of crystallinity. From the above point of view, we attempted to elucidate the relationship between the mechanical and thermal properties and the crosslink density of a PVA-borax complex. A completely saponificated and salt-free PVA was used and its viscosity-average molecular weight was about $8.8 \times 10^{4}$. The crosslinking reagent was a commercially supplied purified sodium tetraborate, which was used without further purification. Gels were prepared by mixing $20 \mathrm{ml}$ of 1 base-mol/l PVA solution with $10 \mathrm{~m} l$ of sodium tetraborate solutions $\left(1 \times 10^{-1}\right.$ $\left.1 \times 10^{-4} \mathrm{~mol} / l\right)$, and then, to obtain dried films, spread out onto a cleaned Acrylite plate (Mitsubishi) floating on a mercury surface. By assuming that all the borate ions added react with the $\mathrm{OH}$ groups of PVA as a tetrafunctional crosslinking reagent and form 1:4 complexes even in the dried gel, ${ }^{8}$ the degree of crosslinking can be written as a ratio of $\left[(\mathrm{VA})_{4} \mathrm{~B}^{-}\right] /[\mathrm{VA}]$. Here, $\left[(\mathrm{VA})_{4} \mathrm{~B}^{-}\right]$and [VA] represent the numbers of moles of the 1:4 complex and the polymer based on its repeating units, respectively. Although the crosslink density determined thermodynamically should generally be used, the above mentioned ratio was used due to the difficulty of obtaining equilibrium stress and/or swelling measurements on these samples. A completely dried film was used for the measurements. The temperature dependency of the mechanical parameters $\left(E^{\prime}\right.$ and $\left.\tan \delta\right)$ at $20 \mathrm{~Hz}$ was obtained by using a viscoelastic spectrometer (Iwamoto). DTA and TG measurements were run on a Rigakudenki-8001 Thermoflex with the heating rate of $10^{\circ} \mathrm{C} / \mathrm{min}$.

Some typical DTA curves for the variously crosslinked PVA's are shown in Figure 1. A 


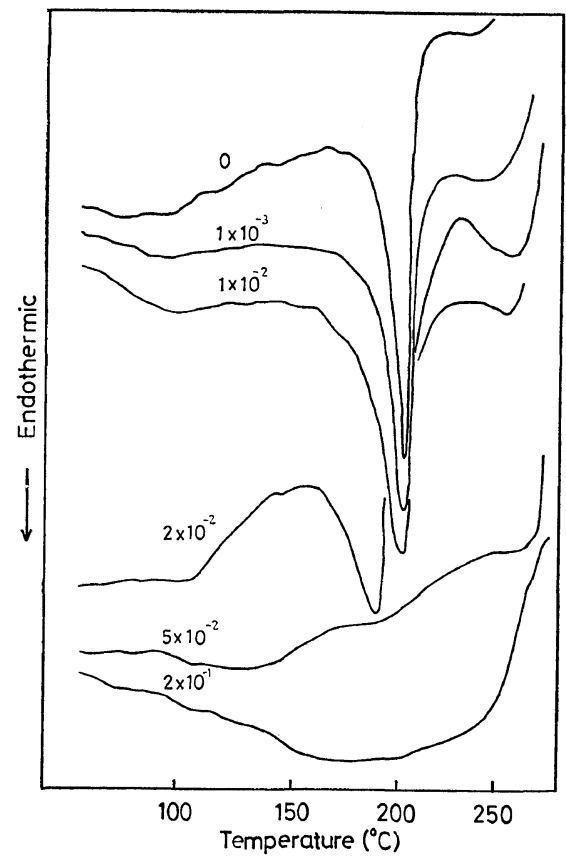

Figure 1. DTA curves for the variously crosslinked PVA. $\left[(\mathrm{VA})_{4} \mathrm{~B}^{-}\right] /[\mathrm{VA}]$ is shown for each curve.

sharp endothermic peak at about $200^{\circ} \mathrm{C}$ is undoubtedly due to the fusion of the crystalline phase of PVA. This peak was broadened and shifted to the low temperature side by introduction of crosslinks, reflecting the destruction of the crystalline structure. For the highly crosslinked samples $\left(>3 \times 10^{-2}\right)$, the peak disappeared. This means that the crystallization of PVA chains is arrested completely judging from the DTA point of view, and the polymer chains would be then in an essentially amorphous state. Furthermore, although it is not shown in the figure, the TG curves did not exhibit such weight loss as could be ascribed to polymer degradation or to dehydration of the unreacted borax up to the temperatures of fusion or up to about $230^{\circ} \mathrm{C}$ for the highly crosslinked samples. It seems therefore that the PVA-borax complex is fairly stable up to relatively higher temperatures.

Figures 2 and 3 show the temperature dependences of $E^{\prime}$ and $\tan \delta$, respectively. In these figures, as the crosslinking increases the temperature dependency of the dynamic parameters changes from those typical of a semicrystalline polymer to those of an amorphous polymer.

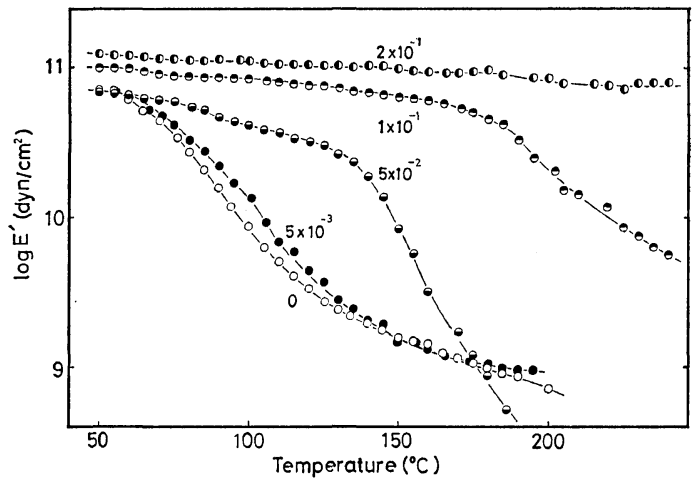

Figure 2. Temperature dependence of storage modulus, $E^{\prime}$, for the variously crosslinked PVA. $\left[(\mathrm{VA})_{4} \mathrm{~B}^{-}\right] /[\mathrm{VA}]$ is shown for each curve.

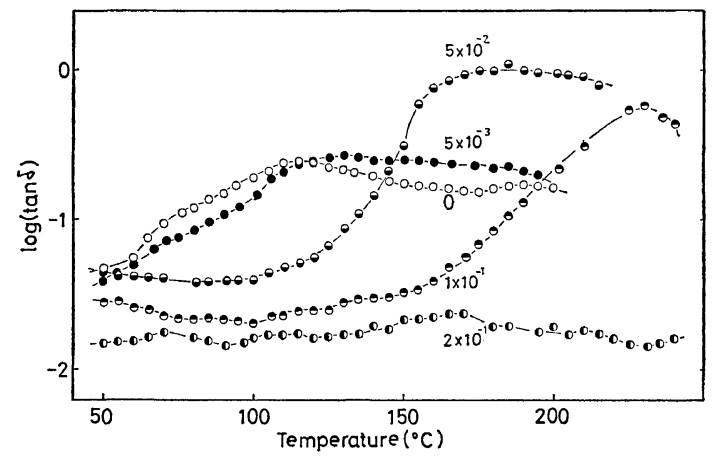

Figure 3. Temperature dependence of $\tan \delta$ for the same samples as shown in Figure 2.

The glass-rubber transition region and the $\alpha_{\mathrm{a}}$ loss peak were shifted to higher temperatures with increasing crosslink density. For the most highly crosslinked sample, such a transition could not be observed and the modulus remained very high in the temperature range examined. In other words, such a sample is essentially a polymeric glass up to the decomposition temperature. The crystalline absorptions, $\alpha_{\mathrm{c}}$ and $\beta_{c}$, (which are generally ascribed to the motions in the perfect and imperfect crystalline phases, respectively) gradually disappeared due to the destruction of crystalline phase, with good correspondence to the DTA curves. The effects of network formation on these absorption temperatures are summarized in Figure 4.

From these results, the large destructive effect of the crosslinking on the crystalline structure and polymeric glass formation suggests that not 
Mechanical and Thermal Properties of Poly(vinyl alcohol) Crosslinked by Borax

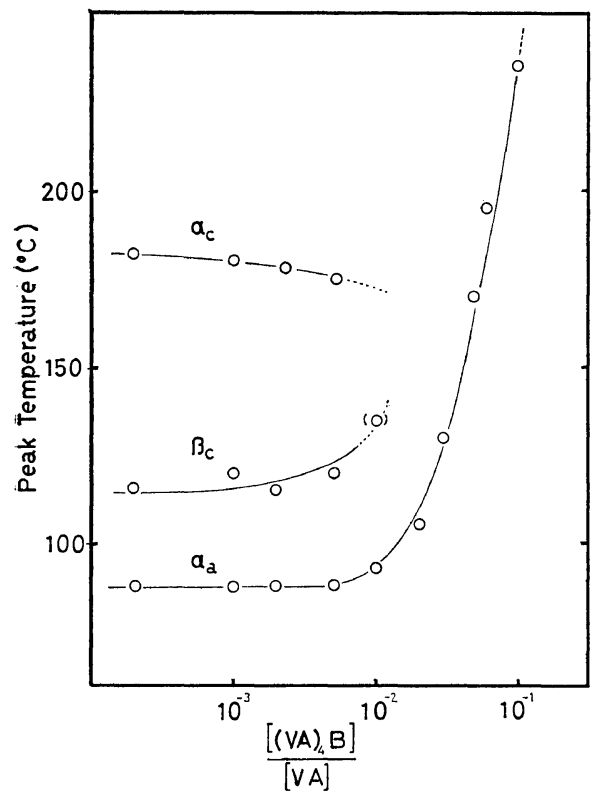

Figure 4. Dependences of the temperatures of each absorption peak ( $\tan \delta_{\max }$ ) on the crosslink density of PVA.

only do the crosslinked units themselves not crystallize but also the neighbouring crystallizable units are prevented for steric reasons from participating in the crystallization process, and that at the same time the crosslinked units greatly immobilize the PVA chains. In addition it can be presumed that, in the course of solidification by solvent evaporation, the chelate formation between the random PVA chains and borate ions has preference for the hydrogen-bond formation between the $\mathrm{OH}$ groups taking part in the crystallization process of PVA chains. From Figure 4, it can be seen that the microBrownian motions of amorphous chain segments begin to significantly retarded in the vicinity of $\left[(\mathrm{VA})_{4} \mathrm{~B}^{-}\right] /[\mathrm{VA}]=2 \times 10^{-2}$. This value of $2 \times 10^{-2}$ implies that there is one $\mathrm{OH}$ group reacted with each borate ion per 12.5 free $\mathrm{OH}$ groups. Thus, if a random crosslinking occurred, the number of carbon atoms between crosslinking points would be about 25. Alternatively, if borate ions could react with only two sets of adjacent two $\mathrm{OH}$ groups, the number would be about 50. These estimated values seem to be reasonable ones for the length of segment undergoing ordinal micro-Brownian motions. ${ }^{9}$ Furthermore, the disappearance of the glass-rubber transition for the most highly crosslinked sample can be ascribed to the immobilization of chain segments by the excessive subdividing of the molecular chains.

\section{REFERENCES}

1. H. Deuel and H. Neukom, Makromol. Chem., 3, 13 (1949).

2. S. Saito, H. Okuyama, H. Kishimoto, and Y. Fujishima, Kolloid-Z., 144, 41 (1955).

3. G. L. Roy, A. L. Laferriere, and J. O. Edwards, J. Inorg. Nucl. Chem., 4, 106 (1957).

4. R. E. Schchat and L. Z. Raymond, "Advances in Chemistry Series," No. 25, American Chemical Society, Washington, D.C., 1960.

5. K. Bolewski and B. Rychly, Kolloid-Z. Z. Polym., 228, 48 (1968).

6. R. K. Schultz and R. R. Myeres, Macromolecules, 2, 281 (1969).

7. L. Mandelkern, "Crystallization of Polymers," McGraw-Hill, New York, N.Y., 1964, Chap. 6.

8. N. Okada and I. Sakurada, Kobunshi Kagaku (Chem. High Polymers), 15, 23 (1958).

9. D. Katz and A. Tobolsky, J. Polym. Sci., Part $A, 2,1595$ (1964). 